\title{
ESRA, AN EDUCATIONAL SOFTWARE FOR INTRODUCING STOCHASTIC SCHEDULING TO CIVIL ENGINEERING STUDENTS
}

\author{
Jorge Salas ${ }^{1}$, Leonardo Sierra², Víctor Yepes ${ }^{3}$ \\ ${ }^{1}$ Universitat Politècnica de València (SPAIN) \\ ${ }^{2}$ Universidad de La Frontera (CHILE) \\ ${ }^{3}$ ICITECH, Universitat Politècnica de València (SPAIN)
}

\begin{abstract}
Classical scheduling techniques are commonly employed tools in civil engineering schools worldwide for teaching project planning and management. Techniques such as the Critical Path Method (CPM), Precedence diagram method (PDM), Gantt chart or the Program Evaluation and Review Technique (PERT) present the advantage of their simplicity, ease of understanding and that they are implemented in the most accepted project management software such as Ms Project or Primavera P6. These scheduling techniques, however, present important limitations in dealing with the uncertainty inherent to construction project management. On the one hand, the deterministic approach of CPM to project planning learning reduces sensitivity and understanding of the factors that significantly alter and challenge the success of a project, while on the other hand PERT shows too limited capabilities in uncertainty modelling and underestimates the project duration standard deviation.
\end{abstract}

Schedule Risk Analysis (SRA) is a stochastic method that has been pointed out to promote that students start managing projects more effectively and more efficiently. In this study, we employ an educational SRA software (ESRA) to help students understand the underlying assumption of stochastic scheduling, as well as to make explicit the advantages of stochastic scheduling compared to classical methods such as CPM or PERT. ESRA affords modelling both the uncertainty in the duration of activities, and the relationship between these uncertainties, expanding the range of planning problems, which students can now assess. This research was implemented in four stages through a workshop. First, we introduced the theoretical foundations of Monte Carlo Simulation, the method underlying most uncertainty assessment methods. Second students employed ESRA to see how this method works. Third, students worked around a case-study of the construction projects management and analysed the results, comparing those from the stochastic assessment with those of the deterministic assessment. Finally, they were asked to respond a questionnaire in which they had to address real-world decision-making regarding project scheduling that required having project uncertainties into account.

Keywords: Stochastic scheduling, Schedule Risk Analysis, Monte Carlo Simulation, Civil engineering, Project planning.

\section{INTRODUCTION}

The ability to plan and schedule civil works is a key competence for civil engineering graduates worldwide [1, 2], which attainment has been traditionally addressed in civil engineering schools by training students into classical scheduling methods such as the Critical Path Method (CPM), Precedence diagram method (PDM), Gantt chart or the Program Evaluation and Review Technique (PERT) [3]. These methods provide a logical mathematical framework that is used to structure project scheduling by first defining tasks or activities, and then by determining the sequence of these activities in order calculate, based on the tasks' durations, the project's total duration. They have the advantage of being easy to understand thanks to their simplicity, and of being implemented in the most accepted project management software such as Microsoft Project or Primavera P6. But at the same time, these techniques have the inconvenience of being inadequate for dealing with the uncertainty inherent to construction project management $[3,4]$ CPM, on the one hand, employs a purely deterministic approach for estimating project duration, which utterly prevents it from accounting for any form of uncertainty. PERT, on the other hand, affords planners to define tasks duration through a 3-point estimation (likely, pessimistic and optimistic durations), but this limited capability for uncertainty modelling, together with the severe underestimation of the project duration standard deviation in which PERT incurs [3], render also this method as inappropriate for addressing construction project uncertainties. 
This lack of capacity for uncertainty modelling and analysing in the methodologies typically taught in civil engineering schools for project scheduling, must be one of the reasons why although construction risks have attracted increasing concerns for many years, the construction industry has a poor reputation in risk analysis [4, 5]. Usually, construction practitioners have relied on unsystematic approaches, such as intuition and in-house techniques, for analysing and evaluating project uncertainties [5, 6]. This unsatisfying practical reality reflects the lack of proper risk management capability in construction practitioners [4], and reveals a gap in the programming and risk management capabilities of students and practitioners [7] that educators must help to bridge [3, 4].

To help bridging this gap, this study resorts to Schedule Risk Analysis (SRA) for first rising students' awareness on the effect of project uncertainties over project duration, then for providing them with a basic training in modelling and evaluating scheduling uncertainties. SRA is a stochastic scheduling method that incorporates project tasks uncertainties into project duration evaluation, which has been already suggested for introducing students intro a more effective and efficient way of managing construction projects [3]. In our study we employ ESRA [8], an educational SRA software, to help students understand the underlying assumption of stochastic scheduling, as well as to make explicit the advantages of stochastic scheduling compared to classical methods such as CPM or PERT. ESRA affords modelling both the uncertainty in the duration of activities, and the relationship between these uncertainties, expanding the range of planning problems that students can now assess.

\section{METHODOLOGY}

For this research, a four-stage workshop was designed in order to introduce SRA to students, facilitate their understanding of the SRA foundations, and to provide them with the basics to model projects in SRA, and to interpret SRA results and employ them for risk management purposes. The workshop was held with graduate students from the Construction Engineering and Civil Engineering careers at the University of La Frontera - Chile. In the first stage of the workshop, students were provided with an introduction to SRA in which this method was contextualized within the field of risk analysis and management, and its theoretical foundations presented. In this stage, we emphasized the role that Monte Carlo Simulation (MCS) plays in probabilistic analysis, employing ESRA to support theory with a visual illustration of how MCS works. MCS is a technique employed for probabilistic analysis in many fields related to civil engineering such as infrastructure and urban planning [5], [9-14], and therefore the understanding of its theoretical foundations is a valuable knowledge that students can connect with uncertainty analysis methods in areas other than project scheduling. Second, students worked around a case study in which the schedule of a mock construction project was modelled and evaluated through ESRA. Third, they were guided throughout the analysis of the results to extract relevant knowledge for construction management, and to compare them with those of the deterministic assessment to make explicit the differences between stochastic scheduling and classical scheduling techniques. Finally, for assessing the acquired knowledge we employed a questionnaire composed of 11 open questions, grouped into two categories, which the students had to complete.

\section{RESULTS}

\subsection{Workshop module I: Understanding the principles of SRA}

\subsubsection{Theoretical foundations of risk analysis and stochastic scheduling}

SRA is a quantitative uncertainty analysis technique that seeks to connect the risk information of project activities to the baseline (probabilistic) schedule, in order to assess the potential impact of project uncertainty on its duration, as well as to provide information on the effect of uncertainty of individual project tasks/activities on project duration. In the case, this effect is unfavourable (e.g. increased duration), it will produce risk, while in the case of being favourable (e.g. reduced duration) it will produce an opportunity.

SRA is an extension of the CPM method in which activity durations are no longer represented as a certain number of time units, but as a random (stochastic) variable. These random variables depict the uncertainty in tasks durations, and they should be set up by the planner. SRA's framework, therefore, can be formalized as a stochastic graph in which project activities are represented by nodes, connected between them by arcs representing the precedence diagram method rules (Finish-Start, Start-Start, Start-Finish, Finish-Finish), whose potential durations are represented by a given 
probability distribution instead of by a number representing a certain amount of time units (as in the case of CPM).

These stochastic variables representing tasks uncertain durations can be somehow connected between if, for example, they share a common source of uncertainty, i.e., the cause producing uncertainty is affecting several tasks (e.g. worse than expected ground conditions affecting different excavation stages). In stochastic programming, this connection between events (ground conditions different from expected) is modelled by correlating the affected stochastic variables. This correlation ranges between -1 when there is total inverse correlation (if one variable increases, the other variable always decrease) to 0 when there is no correlation (there is no connection between variables), to +1 when there is total direct correlation (if one variable increases, the other variable always increases).
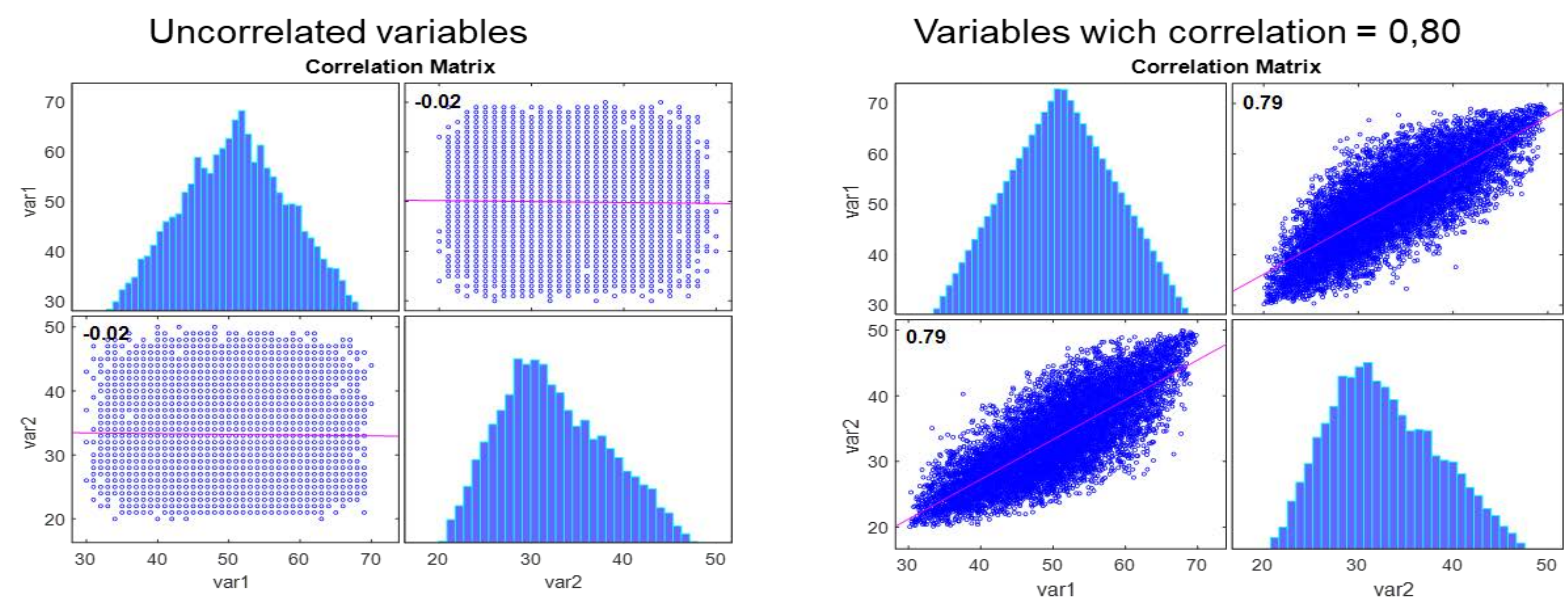

Figure 1. Correlation matrices (histograms and scatter plots) uncorrelated and correlated variables

For the assessment of the project duration, SRA commonly relies on the Monte Carlo Simulation (MCS) technique. This method builds upon the assumption of the Law of the large numbers, which states that for a sufficiently large number of observations (simulations) of an event (project end), there is a very high probability that the average of the observations (empirical average) is very close to the expected value of the event being observed. The implications of this law are very important since it affords avoiding the analytical formulation of the probabilistic model representing the project schedule, which is an extremely unpractical labour. In this approach, the project duration is calculated as the mathematical expectation of the stochastic variable (the function) representing the project schedule, which is in turn formulated based on the stochastic variables (PDFs) representing the project's tasks durations (Figure 2,Figure 3). While this approach is very inconvenient even for the simpler projects, it becomes completely unpractical for real-word projects that usually include a high number of activities linked by a variety of types of relationships.

Project consisting in 2 successive tasks :

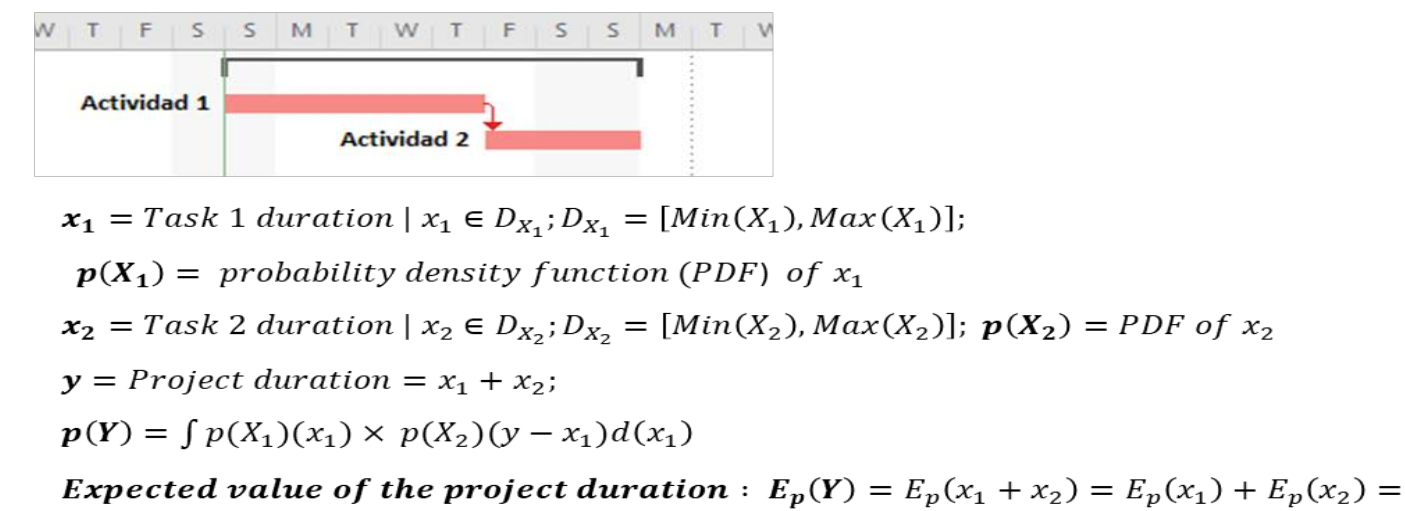

$$
\int_{x_{1} \in D_{X_{2}}} x_{1} p\left(x_{1}\right) d x_{1}+\int_{x_{2} \in D_{X_{2}}} x_{2} p\left(x_{2}\right) d x_{2}
$$

Figure 2. Example of analytical formulation of the simplest case of a schedule with stochastic durations. 
Project consisting in 2 succesive tasks and another one starting from the begining:

a) $x_{1}+x_{2} \geq x_{3}$

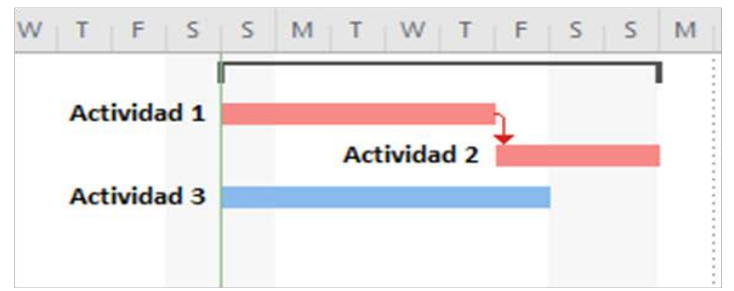

b) $x_{1}+x_{2}<x_{3}$

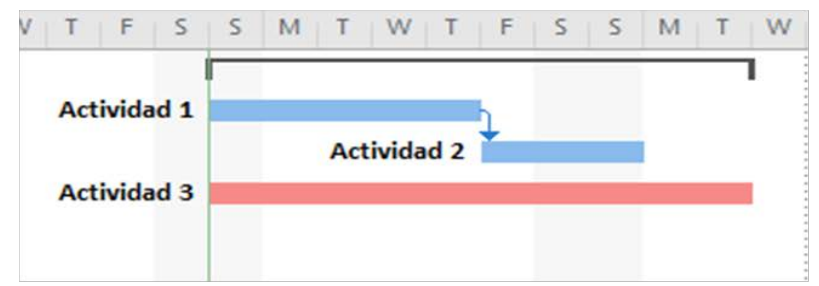

- Expected value of the project duration: $\boldsymbol{E}_{\boldsymbol{p}}(\boldsymbol{Y})=\left\{\begin{array}{l}\text { a) } E_{p}\left(x_{1}+x_{2}\right) \text { when } x_{1}+x_{2} \geq x_{3} \\ b) E_{p}\left(x_{3}\right) \text { when } x_{1}+x_{2}<x_{3}\end{array}\right.$

Figure 3. Example of analytical formulation of a three tasks schedule with stochastic durations

\subsubsection{Visual illustration of the principles underlying Monte Carlo Simulation}

In contrast with the difficulties entailed by the analytical approach, MCS affords using the CPM logic as a simulator to obtain a number of simulations sufficient to produce an acceptable estimate of the project duration. By assigning to each task duration values randomly extracted from their representative PDF, MCS builds inputs for each simulation and thus affords calculating a set of project durations (simulation results) which can be further statistically analysed in order to obtain enriched information. For illustrating how MCS works, we resorted to ESRA, an SRA software specifically designed to introduce this technique to planners familiar with classical scheduling techniques but unfamiliar with stochastic scheduling. Once introduced the project's scheduling logic and duration uncertainties, ESRA displays the results of the MCS results as the simulations are being performed, which affords observing two features of MCS that confirms the postulates of the Law of the large numbers (Figure 4):

- First, ESRA shows how as the number of simulations increase, the mean of their results stabilize, i.e., the different between the means of simulations in the last one with that of the previous one tends to zero.

- Second, ESRA shows how as the number of simulations increase, the standard deviation of the mean of the simulation results decrease.

This way, students get a visual illustration on the functioning of the MCS technique, which is helpful in the understanding of its theoretical foundations.

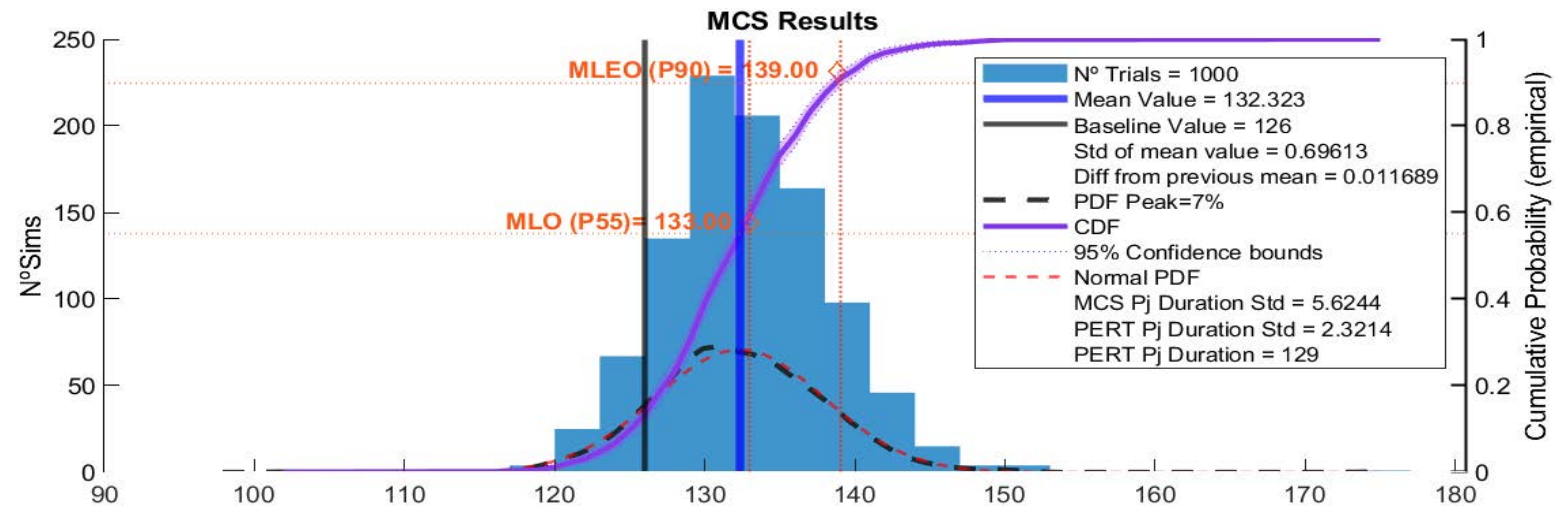

Figure 4. Information displayed by ESRA during the evaluation of simulations. The legend "Diff from previous mean" refers to the difference between the current and the previous mean of the simulations performed, while the legend "Std of mean value" refers to the standard deviation of the series of mean values of the simulations performed.

Along with the information regarding the results of the simulation process, ESRA also facilitates the risk and the opportunity of deviating from the baseline duration. Risk can be defined as a potential realization of an undesired situation, and it has been usually formalized as the product of the probability of occurrence times the consequences of such realization (impact) [11]. This formalization 
is in reality a transposition to mathematical language of its linguistic enunciation, since the expression of risk as the product of probability and impact is the expected value (mathematical expectation) of an undesired outcome (worst project performance).

Risk: Expected value (mathematical expectation) of an undesired outcome (damage).

- $y=$ project duration

- $y_{\text {Base }}=$ project baseline duration

- $i=$ impact $=y-y_{\text {Base }}$

- Damage $=\left\{\begin{array}{l}i \text { when } i>0 \\ 0 \text { when } i \leq 0\end{array}\right.$

- $\boldsymbol{R i s k}=\boldsymbol{E}[\operatorname{Damage}(\boldsymbol{y})]=(i \times p(i>0))+(0 \times(1-p(i>0)))$

- Risk $=i \times p(i>0)=$ impact $x$ probablity of Damage

Figure 5. Formulation of risk as the product of probability of occurrence and impact

Again, the analytical formulation of this becomes a complicated task that is alleviated thanks to MCS. As in the case of the evaluation of simulations, ESRA facilitates the understanding of the mathematical

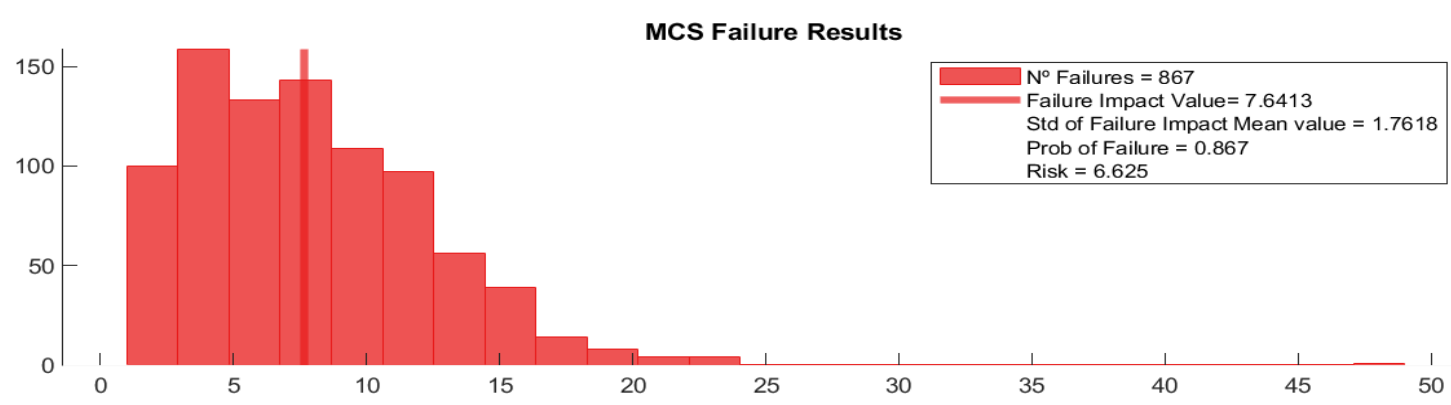

Figure 6. Results of the simulations that produced a damage (increase in project duration), and calculation of the probability, impact and risk of suffering damage

Conversely to the risk, the opportunity is calculated based on the realizations improving project performance, i.e., reducing project duration (Figure 7).

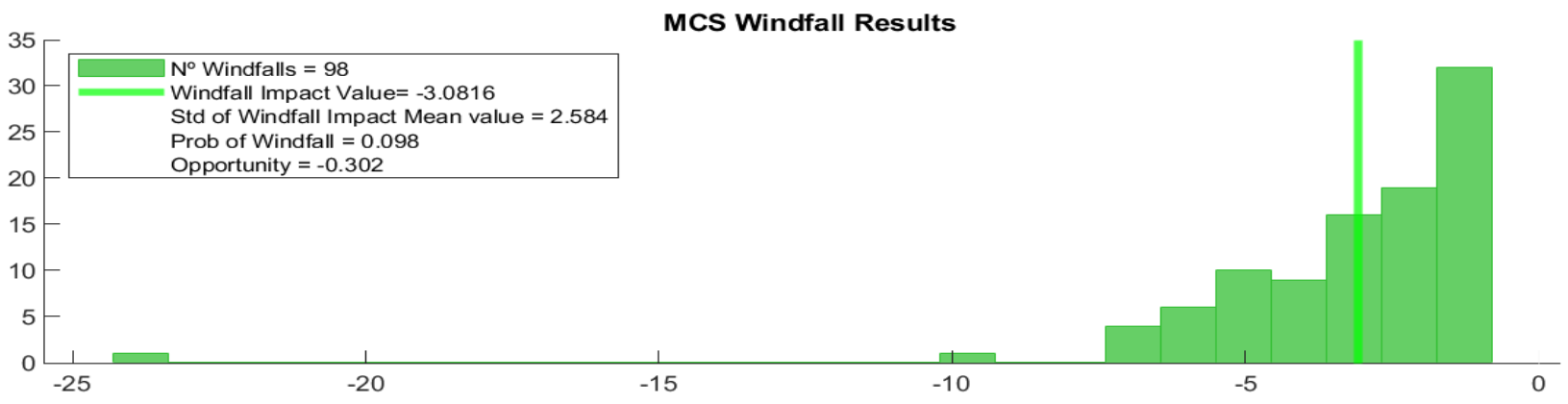

Figure 7. Results of the simulations that produced a windfall (reduction of project duration), and calculation of the opportunity

\subsection{Workshop module II: Modelling a project in SRA}

\subsubsection{Presentation and deterministic modelling of the case study}

The case study consists in the construction of a 5-floors building's reinforced concrete structure (Figure 8), and it comprises the following activities:

- Earthworks (Task 1): it consists of excavating a plot of land with a medium excavation difficulty up to a depth of $3.5 \mathrm{~m}$. 
- Foundation (Task 2): it is of the semi-deep type, and its level of support is expected at a depth of 2.5 from the casting level, although it could reach $5 \mathrm{~m}$ at some points.

- Structure: includes the construction of pillars and slabs (2 tasks by floor) for each floor, which are divided into two areas, separated from each other by a structural joint, and carried out by independent teams of workers:

- Zone A (Tasks $4,5,8,9,12,13,16,17,20,21$ ): It is the largest area, starting four days after starting the basement walls (Task 3 ). The four plants made by this team are carried out sequentially (Finish to start relationships).

○ Zone B (Tasks 6,7,10,11,14,15,18,19,22,23): starts three days before finishing the basement walls. The four plants made by this team are carried out sequentially (Finish to start relationships).

- Roof (Tasks 24,25$)$ : it consists of an accessible flat roof to be built by a company specialized in waterproofing and paving. Tis task begins once the structure of both areas has been completed.

- Suspended sewerage system (Task 26): It can be started once the structure is finished.

This project logic, together with the deterministic estimates of each task's duration, was introduced in ESRA, which yielded the deterministic duration of the project schedule (Figure 8).

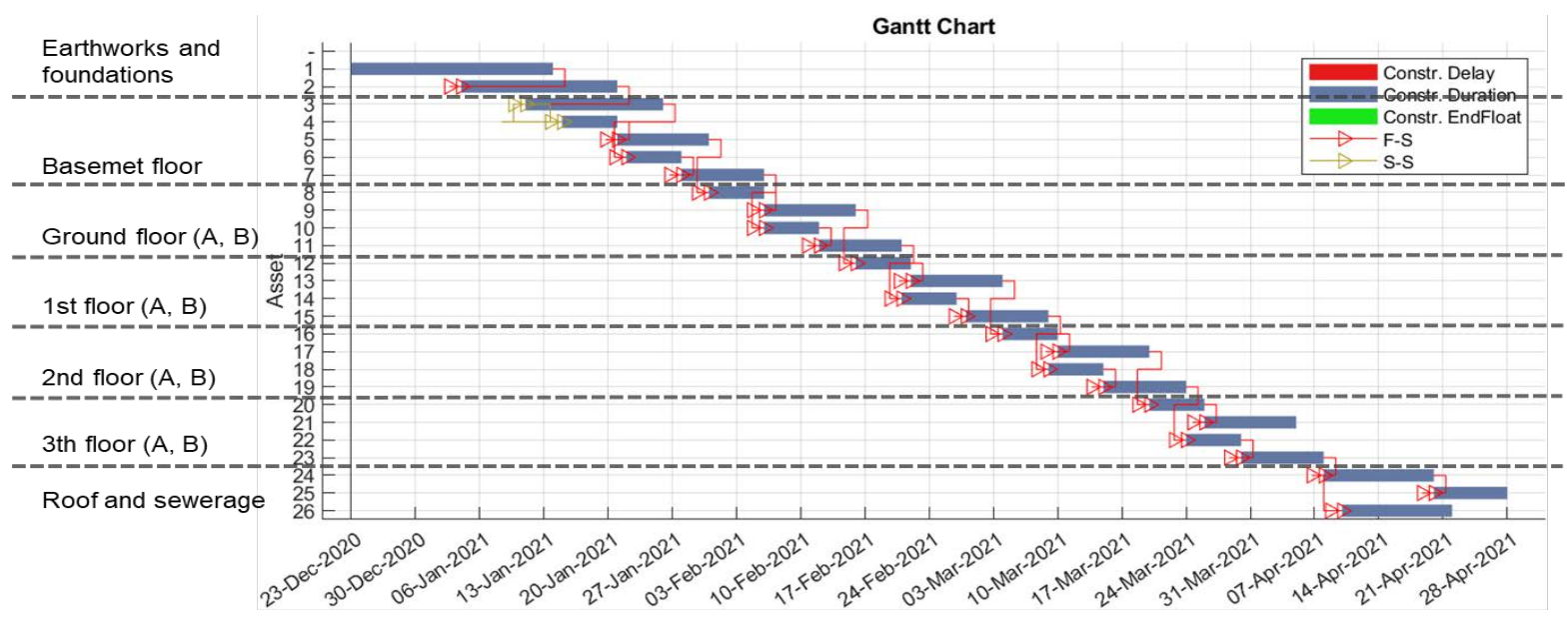

Figure 8. Baseline schedule of the case study facilitated by ESRA. Baseline duration (CPM) is 126 natural days; critical path includes tasks 1, 2, 3, 6, 7, 10, 11, 14, 15, 18, 19, 22, 23, 24, 25

\subsubsection{Stochastic modelling of the case study}

The stochastic modelling of the case study consisted in the setup of two parameters for each task, namely (i) the uncertainty in the task's duration and (ii) the correlation of this uncertainty with that of other tasks.

The configuration of the uncertainty in tasks duration was addressed by assigning the maximum, minimum and most likely duration to each task, and then assigning the correspondent PDF. The first step was resolved based on the teacher's background on construction operations, while for the second step, the following criteria was adopted for the selection of an appropriate PDF:

- High confidence in the estimate of the most likely duration: Beta-PERT distribution (high concentration of realizations around the most likely duration estimate)

- Medium confidence in the estimate of the most likely duration: Triangular distribution (medium concentration of realizations around the most likely duration estimate)

- Low/null confidence in the estimate of the most likely duration: Uniform distribution (no concentration of realizations around the most likely duration estimate)

As to the correlation between tasks' uncertainties, the following guidelines were adopted:

- Strong correlation: sequential tasks, of a very similar nature and affected by common factors (e.g. structure slabs carried out by the same team of workers) 
- Moderate-weak correlation: tasks related to each other and that are developed in parallel even though they do not share characteristics or uncertainty factors (e.g. structure slabs carried out by different teams)

- Weak-null correlation: tasks that are independent of each other, of a different nature, and that share few or no uncertainty factors (e.g. structure slabs and sewerage).

\subsection{Workshop module III: Understanding the results of SRA}

\subsubsection{Results at project level}

Instead of a single project duration, stochastic scheduling yields a set of simulation results describing the project's potential duration. As a consequence, the identification of the project duration in stochastic scheduling implies a decision-making in which the planner should decide which level of confidence the estimation should have, and then identify which simulation result satisfy this requirement. This process is usually articulated by specifying a given percentile of the simulation results cumulative distribution function (CDF) as the desired level of confidence for the project duration estimate [15], and the selection of the corresponding value in the intersection of the desired level of confidence and the simulation results CDF. In ESRA, this project duration estimation is referred to as the most likely outcome (MLO), and its value is calculated accordingly to the percentile $55 \%$ (Figure 4), which was the value setup in the ESRA's corresponding field. The interpretation of this metric is quite straightforward, since it represents the maximum project duration with the probability of the selected percentile. In the case of the $55 \%$ percentile, the MLO is to the right of the median value (percentile 50\%) and therefore represents a duration slightly more conservative than the median which can be taken as the expected project duration (Figure 4, 133 natural days).

Following this logic, ESRA also facilitates the most likely extreme outcome (MLEO), which can be assimilated to a worst-case scenario, by selecting the percentile $90 \%$ [15] in the correspondent field (Figure 4). To illustrate the applicability of this information to real-world decision-making, we employed the MLEO to answer the question of what duration a contractor will accept from which on the he or she will suffer a penalty for delay if the contractor wished to have this probability limited to $10 \%$.

As to the risk and opportunity of completing the project within the baseline duration (126 natural days), ESRA indicated that the risk of delay (6.62 days,Figure 5) heavily outweighs the opportunity of an anticipated end (0.32 natural days, Figure 6$)$, which means that the baseline duration will most surely be exceeded.

\subsubsection{Results at tasks level}

One of the main advantages of SRA compared to classical scheduling methods is the possibility of carrying out a sensitivity analysis. For Satelli [16], the sensitivity analysis is the measure of the effect of input on a particular output, verifying how the uncertainties of the parameters cause uncertainty in the system response. This analysis allows the identification of risk factors in an early stage and is therefore essential for a proactive risk management. ESRA performs two types of analysis: one-attime (OAT) analysis and global sensitivity analysis (GSA).

One-at-a-time sensitivity analysis is one of the simplest yet most common sensitive analysis techniques, in which only one input variable changes in each simulation. This way, it is possible to identify the independent effect of each variable over project performance. In ESRA, tasks correlated to others are evaluated together during OAT to ensure that this analysis properly accounts for the connection between tasks uncertainties. This is the reason why task 7 (basement floor slab, correlated with slabs of floors ground, 1, 2 and 3 ) is more sensitive than task 11 (ground floor slab, correlated with slabs of floors 1, 2 and 3), despite having the same PDF and both being part of the critical path (Figure 8 


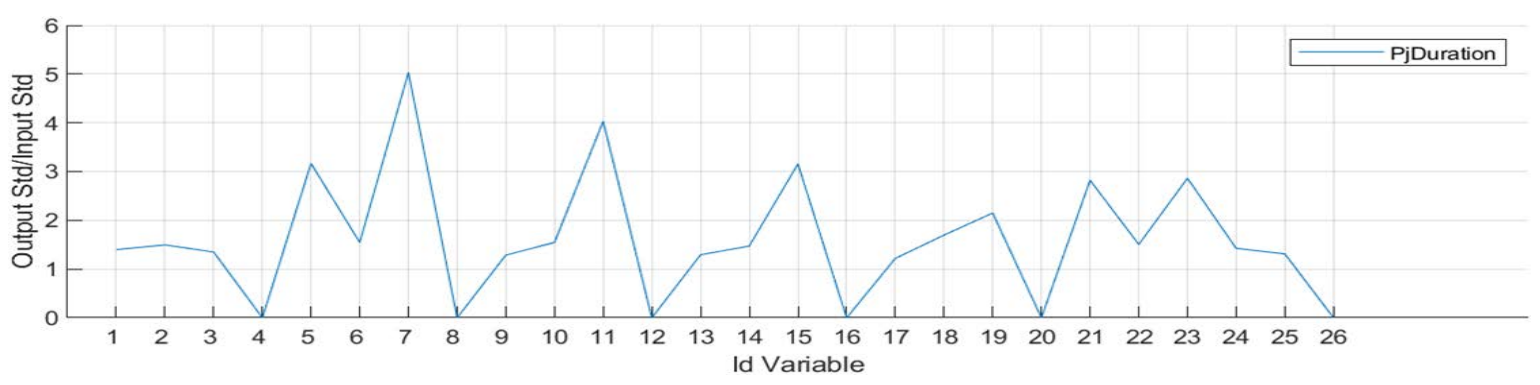

Figure 9. One-at-time results of the case study

One-at-a-time sensitivity analysis, however, cannot account for the combined effect of the input variables, which makes necessary to resort to an additional analysis to grasp this type of effect. Global sensitivity analysis, on the other hand, is a methodology in which all variables are allowed to vary in each simulation and can therefore account for their combined effect [16]. ESRA performs GSA based in the SSCR method, which is a regression-based GSA method, which represents tasks sensitivity through the regression coefficients obtained by considering the values taken by the input variables (tasks) in each simulation and the results of each simulation, as the independent and the dependent variables of a linear regression, respectively. This type of analysis affords the identification of sensitive tasks, like tasks 1 and 2 (Figure 10), that the OAT analysis failed to reveal.

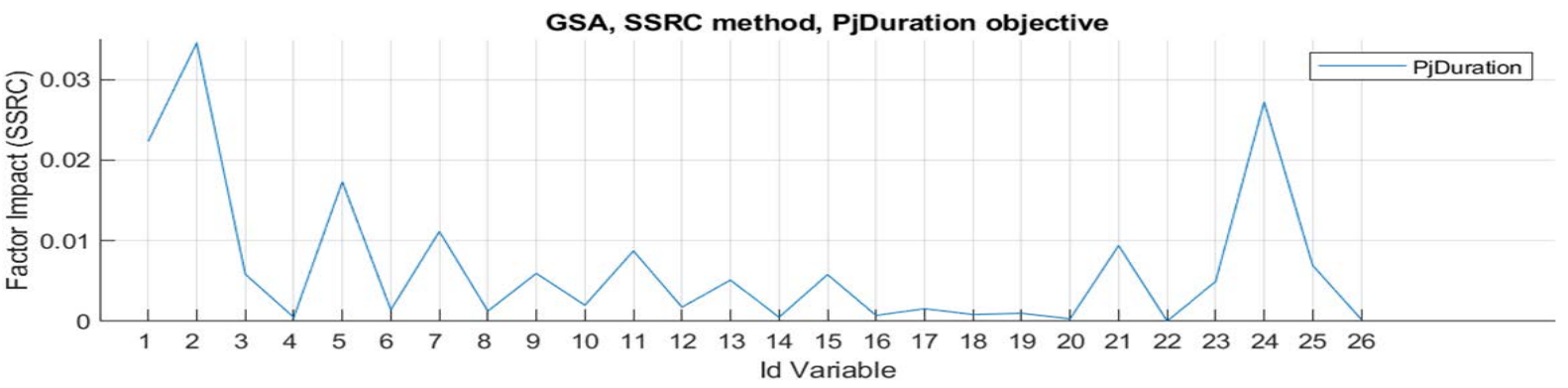

Figure 10. GSA results of the case study

An early identification of risk factors is crucial for an effective risk management. Sensitivity analysis of tasks affords the identification of those activities that require closer control during project execution [17]. ESRA allowed the identification of tasks 5, 7 and 11 through OAT (Figure 9), and of tasks 1, 2, and 24 through GSA (Figure 10), as the most sensitive ones, and therefore as those in which most attention should be paid during construction, and the planning of corrective measures be anticipated.

\subsubsection{Comparison of results from ESRA with those of classical scheduling methods}

As to the differences between CPM and ESRA, on the one hand, the project duration calculated by the former is 7 days shorter than that of the latter (Figure 4), which means a difference of approximately $5.55 \%$. On the other hand, ESRA afforded the identification of the most sensitive tasks, while CPM only affords the set of tasks comprising the critical path, which affords no information on the relative importance of these tasks. It is noteworthy, besides, that CPM was unable to identify task 5 as a risk factor, since it is not part of the deterministic critical path (Figure 8), which illustrate the limitations of deterministic approaches for construction risk management.

As to the differences between PERT and ESRA, on the one hand, the project duration calculated by the former is 6 days shorter than that of the latter (Figure 4), which means a difference of approximately $4.76 \%$. The greatest limitation of PERT, however, is the underestimation of the project duration standard deviation in which PERT incurs [3], which has as practical implications the underestimation of the risk of exceeding a given duration. Figure 4 shows that in the case study, standard deviation of the project duration calculated by PERT and ESRA was 2,32 and 5,62 respectively, i.e. PERT estimation was only $41,28 \%$ of the actual standard deviation. 


\subsection{Workshop module IV: Questionnaire}

\subsubsection{Design of the questionnaire}

Finally, a set of questions was developed to assess to which extent the raising of awareness on the effect of uncertainty on project expected performance, and the acquisition of basic capabilities for performing RSA and connecting RSA results with real-world situations, were achieved throughout the workshop. The questionnaire used is available from the authors by request. The set of questions was classified into two categories that cover the fundamentals of SRA.

The first category focuses in stochastic project scheduling and the interpretation of results at project level, and it includes five questions, while the second category focuses in the interpretation of results at tasks level, and it includes six questions (Table 1).

\subsubsection{Implementation of the workshop and results of the questionnaire}

The workshop took place during the dates 12/28/2020 and 12/29/2020 and it attended 27 students of the school of civil engineering of the Universidad de La Frontera (UFRO), Chile. After the workshop, the questionnaires were completed by the students, which results are shown in Table 1.

In general, the students achieved an acceptable performance in answering the proposed questions, which is indicative of the acquisition of basic competences in stochastic project scheduling and in the understanding of the results of SRA methods. However, the questionnaire revealed difficulties in differentiating between risk and uncertainty (Topic C:2.1, Table 1), as well as in estimating the probability of finishing the project before/after a given date (Topic C:1.4, Table 1).

Table 1. Questionnaire topics per category, and proportion of correct answers

\begin{tabular}{lcc}
\multicolumn{1}{c}{ Topics addressed in the questionnaire } & $\begin{array}{c}\text { Correct } \\
\text { answers (\%) }\end{array}$ \\
\hline Category 1, stochastic project scheduling and results at project level: & 1,2 & $70 \%$ \\
C1.1: Modelling of uncertainty in tasks duration (assignment of PDF) & $30 \%$ \\
C1.2: Modelling of connection between uncertainties of different tasks (assignment \\
of correlation) \\
C1.3: Understanding of the economic consequence of risk of delay (Increase of \\
indirect cost) & 4 & $75 \%$ \\
C1.4: Estimation of the probability of duration beyond a given date, and & 5 \\
understanding of its consequences (probability of penalty) & $40 \%$ \\
Category 2, interpretation of results at tasks level: & \\
C2.1: Connection between sensitivity and uncertainty, and sensitivity and risk & 6,7 \\
C2.2: Identification of the effect of correlation through sensitivity analysis & 8 \\
C2.3: Relation between tasks' sensitivity and membership to the set of tasks within & 9 \\
the critical path & $33 \%$ \\
C2.4: Differences between OAT and GSA & $75 \%$ \\
C2.5: Identification of patterns/relations between tasks through sensitivity analysis & 11 & $70 \%$ \\
\hline
\end{tabular}

\section{CONCLUSIONS}

Classical scheduling methods such as CPM and PERT have been traditionally in civil engineering schools worldwide. These methods, however, are inadequate for dealing to construction projects, which render them useless for risk management purposes. It is necessary, therefore, to train students in advanced scheduling methods, such as RSA, in order to facilitate the acquisition of the risk management capabilities demanded by the construction industry.

This paper describes a methodology for the raising of awareness on the effect of uncertainty on project expected performance, and for the acquisition of basic capabilities for performing RSA and connecting RSA results with real-world situations. The methodology is implemented as a four-stages workshop in which students interact with ESRA, an RSA software specifically designed to introduce this technique to planners familiar with classical scheduling techniques but unfamiliar with stochastic 
scheduling. First, students were provided with an introduction to SRA and its theoretical foundations, along with a visual demonstration of how Monte Carlo Simulation works. Second, students got familiar with the stochastic scheduling of construction project through a case study in which a mock construction project was modelled and evaluated. Third, they were guided throughout the analysis of the results to extract relevant knowledge for construction management, and finally, the students were exposed to a questionnaire for assessing the acquired knowledge.

The results show that the students performed well in most of the topics the questions were about, and that they had difficulties in differentiating between risk and uncertainty, and in estimating the probability of finishing the project before/after a given date. This research sensitizes the civil engineering student with the major role that project uncertainties plays into project duration, showing him/her how stochastic scheduling can be used to inform real-world decision-making.

\section{ACKNOWLEDGEMENTS}

This research was made possible by the support of mgnesio and of the Academic Heads of the Programmes of Career in Construction Engineering and Civil Engineering at the Universidad de La Frontera. The authors are grateful to the students of these programs for taking part in this educational experimentation.

\section{REFERENCES}

[1] F. Eric, G. Vicente, O. Francisco, O. Alexander, S. Álvaro, and A. Pablo, "Application of ProblemBased Learning to Teaching the Critical Path Method," Journal of Professional Issues in Engineering Education and Practice, vol. 141, no. 3, p. 4014016, 2015.

[2] A. Y. Han, A. R. Pearce, and K. Hyuksoo, "Key Competencies for U.S. Construction Graduates: Industry Perspective," Journal of Professional Issues in Engineering Education and Practice, vol. 138, no. 2, pp. 123-130, 2012.

[3] P. Ballesteros-Pérez, G. D. Larsen, and M. C. González-Cruz, "Do projects really end late? On the shortcomings of the classical scheduling techniques," Journal of Technology and Science Education, vol. 8, no. 1, pp. 17-33, 2018.

[4] L. J. Ying, W. Z. P. X., and M. F. Ye, "Does Expectation Match Reality? Examination of Risk Management Education in China," Journal of Technology and Science Education, vol. 140, no. 3, p. 4014002, 2014.

[5] L.A. Sierra, V. Yepes, E. Pellicer, "Assessing the social sustainability contribution of an infrastructure project under conditions of uncertainty", Environmental Impact Assessment Review, 67, 61-72, 2017.

[6] E. Pellicer, L. A. Sierra, V. Yepes, "Appraisal of infrastructure sustainability by graduate students using an active-learning method," Journal of Cleaner Production, vol. 113, pp. 884-896, 2016.

[7] W. Peng, F. Yingbin, P. Joshua, and Z. Yun, "Educational Attainment and Job Requirements: Exploring the Gaps for Construction Graduates in Australia from an Industry Point of View," Journal of Professional Issues in Engineering Education and Practice, vol. 141, no. 4, p. 6015001, Oct. 2015.

[8] Educational Software, Accessed 28 December, 2020, Retrieved from https://www.mgnesio.es/servicios/software-educativo

[9] J. Salas, V. Yepes, "A discursive, many-objective approach for selecting more-evolved urban vulnerability assessment models," Journal of Cleaner Production, vol. 176, pp. 1231-1244, 2018.

[10] J. Salas, V. Yepes, "Enhancing Sustainability and Resilience through Multi-Level Infrastructure Planning," International Journal of Environmental Research and Public Health, vol. 17, no. 3, p. 962, 2020.

[11] J. Salas, V. Yepes, "MS-ReRO and D-ROSE methods: Assessing relational uncertainty and evaluating scenarios' risks and opportunities on multi-scale infrastructure systems," Journal of Cleaner Production, vol. 216, pp. 607-623, 2019. 
[12] T. García-Segura, V. Yepes, D.M. Frangopol, "Multi-Objective Design of Post-Tensioned Concrete Road Bridges Using Artificial Neural Networks", Structural and Multidisciplinary Optimization, vol. 56, no. 1, pp. 139-150, 2017.

[13] C. Torres-Machi, A. Chamorro, E. Pellicer, V. Yepes, C. Videla, "Sustainable Pavement Management Integrating Economic, Technical, and Environmental Aspects in Decision Making", Transportation Research Record, vol. 2523, pp. 56-63, 2015.

[14] J. Salas and V. Yepes, "VisualUVAM: A Decision Support System Addressing the Curse of Dimensionality for the Multi-Scale Assessment of Urban Vulnerability in Spain," Sustainability, vol. 11, no. 8, p. 2191, Apr. 2019.

[15] B. G. A., "Probabilistic Estimation and Allocation of Project Time Contingency," Journal of Construction Engineering and Management, vol. 137, no. 4, pp. 259-265, Apr. 2011.

[16] A. Saltelli, S. Tarantola, F. Campolongo, M. Ratto, Sensitivity Analysis in Practice. John Wiley \& Sons, 2004.

[17] P. Ballesteros-Pérez, A. Cerezo-Narváez, M. Otero-Mateo, A. Pastor-Fernández, and M. Vanhoucke, "Performance comparison of activity sensitivity metrics in schedule risk analysis," Automation in Construction, vol. 106, p. 102906, 2019. 